\title{
PAPER \\ Fast and Accurate 3-D Imaging Algorithm with Linear Array Antennas for UWB Pulse Radars
}

\author{
Shouhei KIDERA ${ }^{\dagger a)}$, Yusuke KANI ${ }^{\dagger}$, Student Members, Takuya SAKAMOTO ${ }^{\dagger}$, Member, and Toru SATO $^{\dagger}$, Fellow
}

\begin{abstract}
SUMMARY Pulse radars with UWB signals are promising as a highresolution imaging technique that can be used for the non-destructive measurement of surface details in industrial products such as antennas and aircraft. We have already proposed a fast 3-D imaging algorithm, SEABED, that utilizes a reversible transform between the time delay and the target boundary. However, data acquisition is time-consuming when obtaining an accurate image because it assumes a mono-static radar with 2-D scanning of an antenna. In this paper, we utilize linear array antennas and propose a fast and accurate imaging algorithm. We extend the reversible transform for mono-static radars to apply to bi-static radars to reduce the data acquisition time. The effectiveness of the proposed method is verified with numerical simulations and experiments.

key words: UWB pulse radars, fast and accurate imaging, BST, bi-static radar, linear array antennas
\end{abstract}

\section{Introduction}

UWB pulse radars provide high-resolution imaging that is suitable for non-destructive measurement techniques. They can be applied to detect small surface defects on the reflector antennas and bodies of aircraft. Additionally, they are suitable for target locating systems of rescue robots in condition of dark smoke. These applications require realtime imaging. While various imaging algorithms for radars have been proposed, they require intensive computation for target imaging [1]-[4]. Contrarily, we have proposed a fast 3-D imaging algorithm, SEABED, that utilizes a reversible transform BST (Boundary Scattering Transform) between the time delays and the target boundary [5]-[7]. This method provides for fast and non-parametric imaging with the received data. However, it requires a long time for the data-acquisition to obtain an accurate image, because it assumes 2-D scanning of an antenna as a mono-static radar. Thus, there is a trade-off between the data-acquisition time and the accuracy of the image.

In the present paper, we propose fast and accurate imaging algorithm with linear array antennas to resolve this trade-off. The accuracy of the image with the original SEABED is limited to an interval of array antennas because BST is applied only to mono-static radars. The interval of array antennas should be more than half of the center wavelength of the pulse to avoid mutual couplings; this limits the accuracy of SEABED images. In this paper, we extend BST

\footnotetext{
Manuscript received August 13, 2007.

Manuscript revised February 18, 2008.

${ }^{\dagger}$ The authors are with the Department of Communications and Computer Engineering, Graduate School of Informatics, Kyoto University, Kyoto-shi, 606-8501 Japan.

a)E-mail: kidera@aso.cce.i.kyoto-u.ac.jp

DOI: 10.1093/ietcom/e91-b.8.2683
}

to apply to bi-static radars and propose an accurate imaging algorithm. First, we present the shape estimation algorithm in 2-D problems for simplicity. The proposed method is then extended to 3-D problems with linear array antennas. The performance of the algorithm is investigated with numerical simulations and an experiment.

\section{Imaging Algorithm for 2-D Problems}

\subsection{System Model}

The system model is shown in Fig. 1. Here, we deal with 2$\mathrm{D}$ problems and TE mode waves for simplicity. We assume that the target has uniform permittivity and is surrounded by a clear boundary that is composed of smooth curves concatenated at discrete edges. We assume that the propagation speed of the radiowave is constant and known. The induced current at the transmitting antenna is a mono-cycle pulse. We utilize omni-directional antennas and locate them with a fixed interval along the $x$ axis.

$\mathrm{R}$-space is defined as the real space, in which targets and antennas are located, and is expressed by the parameters $(x, z)$. Both $x$ and $z$ are normalized by $\lambda$, which is the center wavelength of the transmitted pulse in the air. We assume $z>0$ for simplicity. The locations of the transmitting and receiving antennas are defined as $(x, z)=\left(X_{\mathrm{T}}, 0\right)$ and $\left(X_{\mathrm{R}}, 0\right)$, respectively. $s^{\prime}\left(X_{\mathrm{T}}, X_{\mathrm{R}}, Z^{\prime}\right)$ is defined as the received electric field. We also define $Z^{\prime}$ with time $t$ and speed of the radio wave $\mathrm{c}$ as $Z^{\prime}=\mathrm{c} t / 2 \lambda . s\left(X_{\mathrm{T}}, X_{\mathrm{R}}, Z^{\prime}\right)$ is defined as the output of the matched filter with the transmitted waveform. We extract the significant peaks of $s\left(X_{\mathrm{T}}, X_{\mathrm{R}}, Z^{\prime}\right)$ as $Z$ for each $X_{\mathrm{T}}$ and $X_{\mathrm{R}}$, and define the extracted surface as $\left(X_{\mathrm{T}}, X_{\mathrm{R}}, Z\right)$, which is called a quasi wavefront. D-space is defined as the space expressed by $\left(X_{\mathrm{T}}, X_{\mathrm{R}}, Z\right)$. The transform from d-space

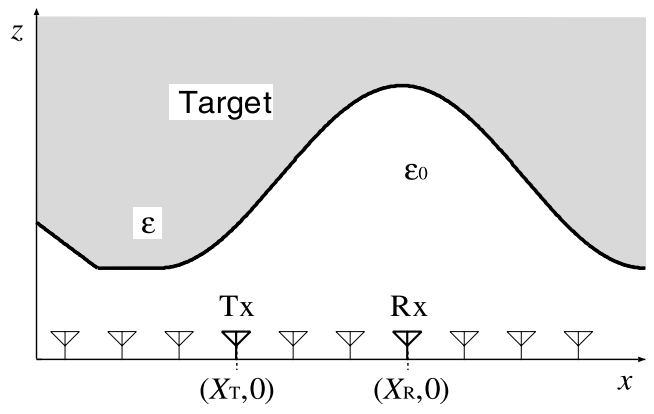

Fig. 1 System model in 2-D problem. 


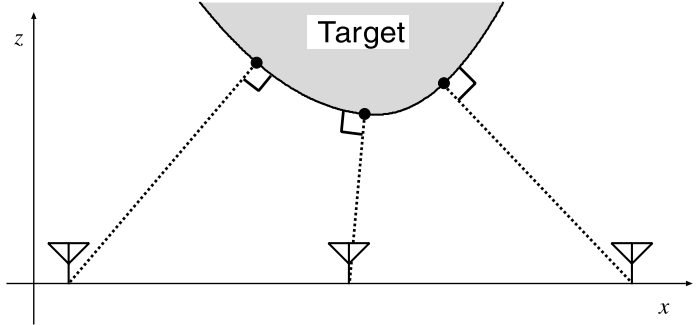

Fig. 2 Relationship between estimated points and antenna locations of the conventional model.

to r-space corresponds to the imaging that we deal with in this paper.

\subsection{Conventional Method}

We have already developed a high-speed imaging algorithm that we term SEABED. This method utilizes a mono-static radar, and defines as $X=X_{\mathrm{T}}=X_{\mathrm{R}}$. It clarifies the existence of a reversible transform BST (Boundary Scattering Transform) between the target boundary $(x, z)$ and the quasi wavefront $(X, Z)[5]$. IBST (Inverse BST) is expressed as

$$
\left.\begin{array}{l}
x=X-Z \partial Z / \partial X \\
z=Z \sqrt{1-(\partial Z / \partial X)^{2}}
\end{array}\right\},
$$

where $|\partial Z / \partial X| \leq 1$ holds. This transform gives us a complete solution for the inverse problem. We extract the part of quasi wavefront as $(X, Z)$ from $\left(X_{\mathrm{T}}, X_{\mathrm{R}}, Z\right)$, where $X=X_{\mathrm{T}}=$ $X_{\mathrm{R}}$ satisfies. A target image is directly estimated by applying the IBST to the quasi wavefront. Although this method realizes a high-speed imaging, the accuracy of the image is limited by the number of the antennas because it assumes a mono-static radar. Here, we define accuracy as the number of the estimated points per unit area of the target boundary. Figure 2 shows the relationship between the estimated points with this method and antenna locations. As shown in Fig. 2, the estimated image has insufficient accuracy. If we increase the number of scanning samples to enhance the accuracy, data acquisition needs long time. Accordingly, there is a trade-off between the time taken to obtain the data and the accuracy of the estimated image.

\subsection{Proposed Method}

To resolve the trade-off described in the previous section, we propose a fast and accurate imaging method with linear array antennas. First, we introduce a reversible transform BST for bi-static radars. We fix the interval of the transmitted and received antennas, and define $d=\left(X_{\mathrm{R}}-X_{\mathrm{T}}\right) / 2 . X$ is defined as $X=\left(X_{\mathrm{T}}+X_{\mathrm{R}}\right) / 2$. The scattering center on the target boundary is defined as $(x, z) . Z$ is the distance to the scattering point, where the law of reflection is satisfied. Figure 3 shows the relationship between the target boundary and a part of the quasi wavefront. With this geometrical condition, $(X, Z)$ is expressed as

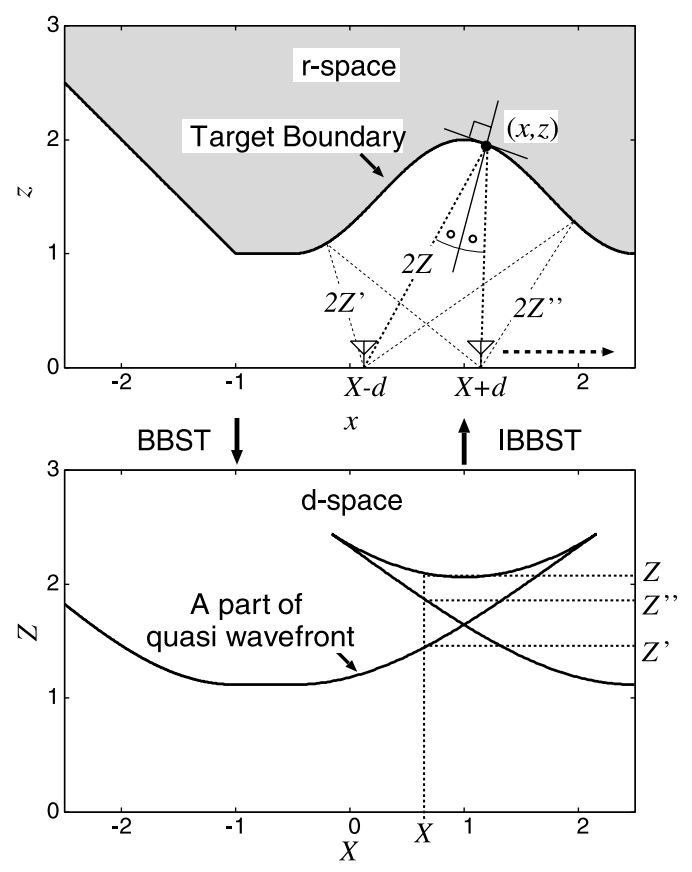

Fig. 3 Relationship between the target boundary and the part of quasi wavefront in bi-static radars.

$$
\left.\begin{array}{l}
X=x+\frac{2 z_{x}\left(z^{2}+d^{2}\right)}{z\left(1-z_{x}^{2}\right)+\sqrt{z^{2}\left(1+z_{x}^{2}\right)^{2}+4 d^{2} z_{x}^{2}}} \\
Z=\sqrt{z^{2}+d^{2}+z z_{x}(X-x)}
\end{array}\right\},
$$

where $z_{x}=\partial z / \partial x$. We call this transform BBST (Bi-static $\operatorname{BST}) .(x, z)$ is also expressed as

$$
\left.\begin{array}{l}
x=X-\frac{2 Z^{3} Z_{X}}{Z^{2}-d^{2}+\sqrt{\left(Z^{2}-d^{2}\right)^{2}+4 d^{2} Z^{2} Z_{X}^{2}}} \\
z=\frac{\sqrt{Z^{2}-d^{2}}}{Z} \sqrt{Z^{2}-(x-X)^{2}}
\end{array}\right\},
$$

where $Z_{X}=\partial Z / \partial X$. We call this transform IBBST (Inverse BBST). The derivations of Eqs. (2) and (3) are given in Appendix A. BBST can be applied to the case $d<0$ because the solutions in Eqs. (2) and (3) do not depend on the sign of $d$. IBBST is effective for real-time imaging, as we can directly estimate the target boundary by using this transform with bi-static radars. We apply IBBST to the linear array antennas as described in the following procedures. We define the number of antennas as $N_{X}$ and the interval of the array antennas as $\Delta X$. First, $k=0$ is set.

Step 1). Apply the matched filter to $s^{\prime}\left(X_{\mathrm{T}}, X_{\mathrm{R}}, Z^{\prime}\right)$, and obtain the output as $s\left(X_{\mathrm{T}}, X_{\mathrm{R}}, Z^{\prime}\right)$.

Step 2). Extract the quasi wavefront as $\left(X_{\mathrm{T}}, X_{\mathrm{R}}, Z\right)$ by connecting the peaks of $s\left(X_{\mathrm{T}}, X_{\mathrm{R}}, Z^{\prime}\right)$.

Step 3. Set $2 d=k \Delta X$ and extract a cross section of the quasi wavefront as $(X, Z)$, where $X=\left(X_{\mathrm{T}}+X_{\mathrm{R}}\right) / 2$ and $X_{\mathrm{R}}=X_{\mathrm{T}}+2 d$ hold. 
Step 4). Apply IBBST to the extracted curve $(X, Z)$ and obtain a target image.

Step 5). Set $k=k+1$. If $k \leq N_{X}-1$ holds, return to Step 3), otherwise complete the shape estimation.

Figure 4 shows Steps 2), 3) and 4) in these procedures. This method enables us to increase the estimated points to $N_{X}\left(N_{X}-1\right) / 2$ by changing the parameter $d$. Figure 5 shows the relationship between the estimated points with the proposed method and antenna locations. Each estimated point is located at a different point on the target surface because

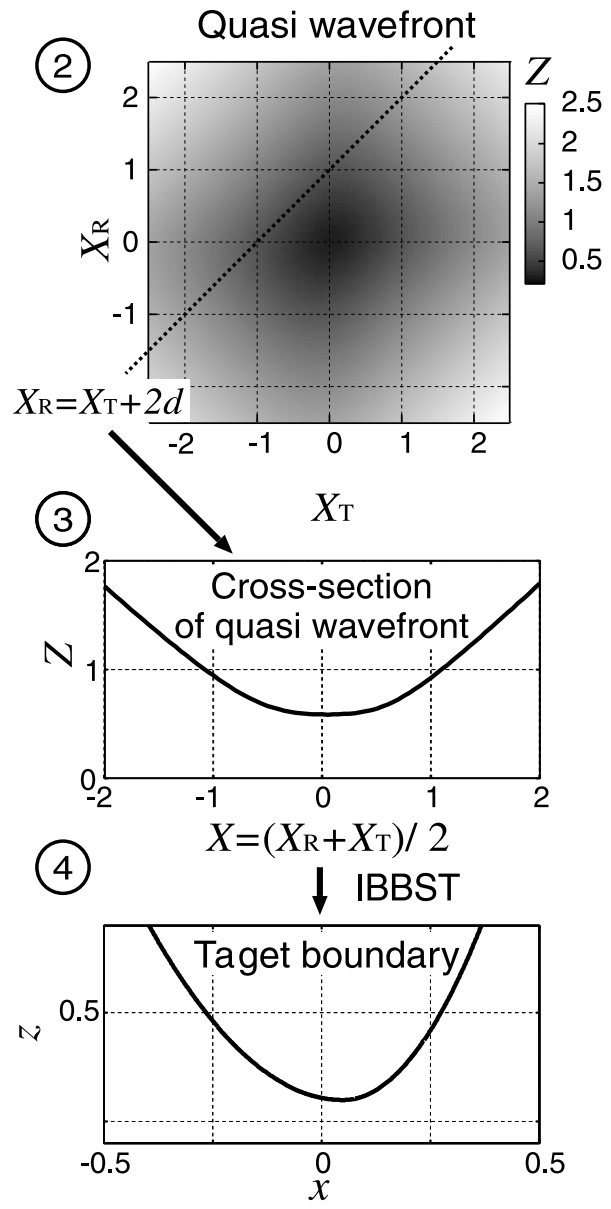

Fig. 4 Quasi wavefront (upper), cross section of the quasi wavefront (middle) and target boundary (lower).

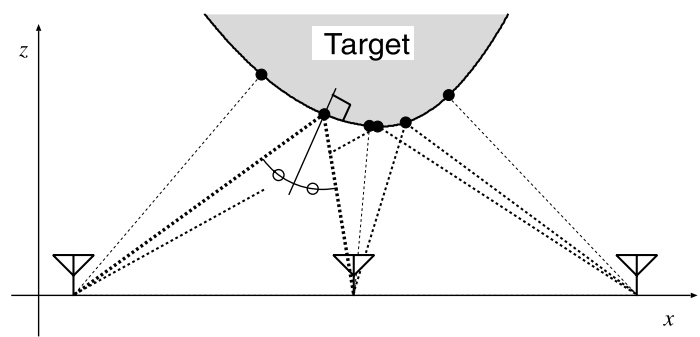

Fig. 5 Relationship between estimated points and antenna locations of the proposed model. the scattered wave propagate along a different path as shown in Fig. 5. Thus, each increased point has an independent information on the target boundary. This means that we can enhance the accuracy of the target image using just a small number of antennas.

\section{Imaging Algorithm for 3-D Problems}

\subsection{Bi-static Radar Imaging for 3-D Problem}

SEABED assumes a mono-static radar in 3-D problems with BST derived in [7]. SEABED is suitable for real-time imaging for 3-D problems. However, it assumes 2-D scanning of the antenna. For the same reasons as those described for 2-D problems, there exists a trade-off between the accuracy and the time taken for data acquisition. To resolve this trade-off, we extend our proposed method to 3-D problems with linear array antennas.

Figure 6 shows the system model for 3-D problems. We set a linear array of antennas along the $x$ axis and scan it along the $y$ axis. The transmitted and received antenna locations are defined in r-space as $\left(X_{\mathrm{T}}, Y, 0\right)$ and $\left(X_{\mathrm{R}}, Y, 0\right)$, respectively. We define the received electric field as $s^{\prime}\left(X_{\mathrm{T}}, X_{\mathrm{R}}, Y, Z^{\prime}\right)$. We define the output of the matched filter with the transmitted waveform as $s\left(X_{\mathrm{T}}, X_{\mathrm{R}}, Y, Z^{\prime}\right)$. The quasi wavefront is extracted as $\left(X_{\mathrm{T}}, X_{\mathrm{R}}, Y, Z\right)$ by connecting the significant peaks of $s\left(X_{\mathrm{T}}, X_{\mathrm{R}}, Y, Z^{\prime}\right)$, and it expresses dspace. $\mathrm{R}$-space is expressed by $(x, y, z)$, which is the target boundary. A transform from $\left(X_{\mathrm{T}}, X_{\mathrm{R}}, Y, Z\right)$ to $(x, y, z)$ corresponds to the imaging.

Let us introduce the reversible transform for bi-static radars for 3-D problems. We set $X=\left(X_{\mathrm{T}}+X_{\mathrm{R}}\right) / 2$ and $X_{\mathrm{R}}=X_{\mathrm{T}}+2 d$, where $d$ is constant. $(x, y, z)$ is defined as the scattering point of the target boundary. $Z$ is the distance to $(x, y, z)$, where the law of reflection is satisfied as shown in Fig. 6. BBST and IBBST in 3-D problems are expressed as

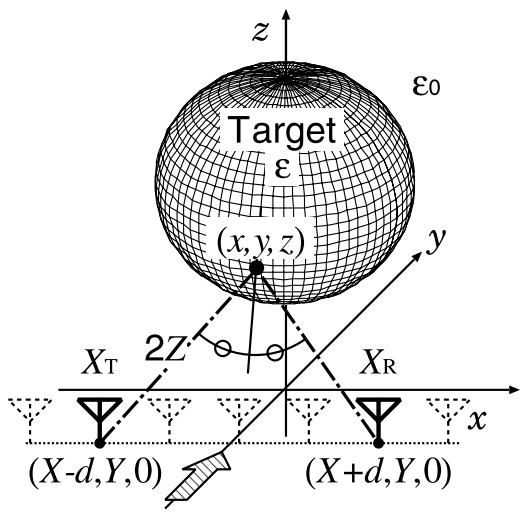

Fig. 6 System model employed for 3-D problems. 


$$
\begin{aligned}
& \left.\begin{array}{l}
X=x+\frac{2 z_{x}\left(z^{2}+z^{2} z_{x}^{2}+d^{2}\right)}{z\left(1-z_{x}^{2}+z_{y}^{2}\right)+\sqrt{z^{2}\left(1+z_{x}^{2}+z_{y}^{2}\right)^{2}+4 d^{2} z_{x}^{2}}} \\
Y=y+z z_{y} \\
Z=\sqrt{z^{2}\left(1+z_{y}^{2}\right)+z z_{x}(X-x)+d^{2}},
\end{array}\right\}, \\
& x=X-\frac{2 Z^{3} Z_{X}}{Z^{2}-d^{2}+\sqrt{\left(Z^{2}-d^{2}\right)^{2}+4 d^{2} Z^{2} Z_{X}^{2}}} \\
& y=Y+Z_{Y}\left\{d^{2}(x-X)^{2}-Z^{4}\right\} / Z^{3} \\
& z=\sqrt{Z^{2}-d^{2}-(y-Y)^{2}-\frac{\left(Z^{2}-d^{2}\right)(x-X)^{2}}{Z^{2}}} .
\end{aligned}
$$

where $z_{y}=\partial z / \partial y$ and $Z_{Y}=\partial Z / \partial Y$ are defined. The derivations of Eqs. (4) and (5) are given in Appendix B. IBBST enables direct estimates of the target shape for 3-D problems.

\subsection{Procedure of the Proposed Method}

The procedure of the proposed method is presented as follows. The point of a quasi wavefront $\left(X_{\mathrm{T}}, X_{\mathrm{R}}, Y, Z\right)$ is extracted by connecting the peak of $s\left(X_{\mathrm{T}}, X_{\mathrm{R}}, Y, Z^{\prime}\right)$. We extract $(X, Y, Z)$ from $\left(X_{\mathrm{T}}, X_{\mathrm{R}}, Y, Z\right)$, where $2 d=k \Delta X$ holds, and $k=0,1, \ldots, N_{X}-1$ is satisfied. IBBST is applied to the extracted wavefront $(X, Y, Z)$ and the target boundary is estimated for each $d$. By changing the distance $d$, we can increase the number of estimated points in the $x$ direction. As with the 2-D problems, each estimated point expresses a different target point. This enables us to enhance the accuracy in the $x$ direction without increasing the number of antennas.

\subsection{Application Example with Numerical Simulations}

We now consider an example of shape estimations using numerical simulations. The target shape is set as shown in Fig. 7. The linear array antenna is set for $-2.0 \lambda \leq x \leq 2.0 \lambda$, where each interval of the antennas is $0.4 \lambda$, and $N_{X}=11$. We scan this array antenna for $-2.0 \lambda \leq y \leq 2.0 \lambda$, where the number of observations $N_{Y}$ is 51 . The interval between the target and array antennas is around $0.7 \lambda$ in minimum. It is assumed that the true quasi wavefront $\left(X_{\mathrm{T}}, X_{\mathrm{R}}, Y, Z\right)$ without noise is given. Figure 8 shows the estimated image with the conventional method, in which the total number of estimated points is 459. It is clear that the conventional method has insufficient accuracy to express the target surface for the $x$ direction, especially on the upper side of the target. This is because the number of estimated points for the $x$ direction is limited to $N_{X}$. In contrast, Fig. 9 shows the estimated image with the proposed method, which achieves more accurate imaging for the $x$ direction, and captures the details on the upper side. In this method, the total number of estimated points is 2754 . This is because the number of the estimated points with the mono-static model is the number

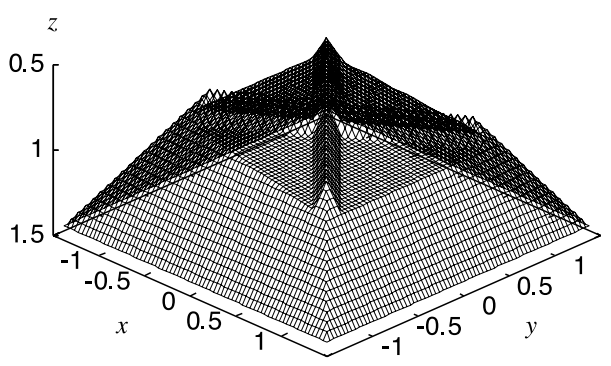

Fig. 7 True target boundary.

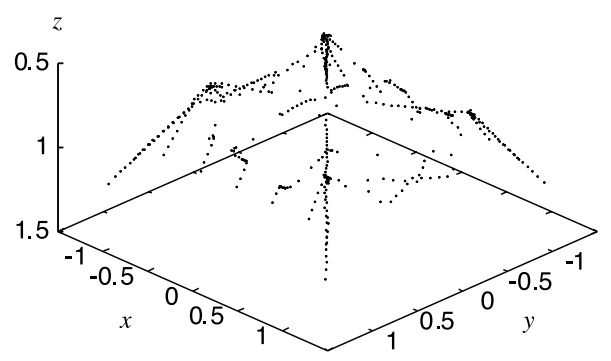

Fig. 8 Estimated image with the conventional method (quasi wavefront is known)

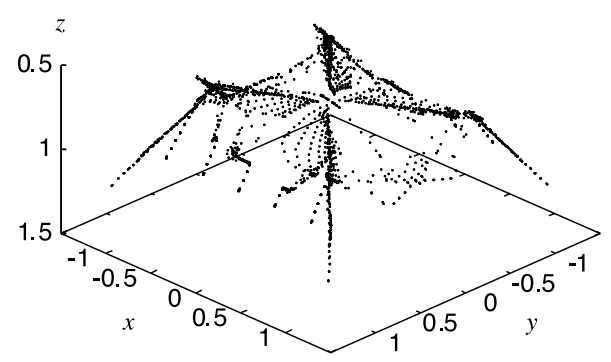

Fig. 9 Estimated image with the proposed method (quasi wavefront is known).

of the scanning samples as $N_{X} \times N_{Y}$; the number of the proposed method increases to the number of combination of transmit and receive antennas. Thus, the number of the estimated points becomes $N_{X}\left(N_{X}-1\right) / 2 \times N_{Y}$, where we remove the end points of the quasi wavefront because the derivatives $\partial Z / \partial X$ and $\partial Z / \partial Y$ are not correctly calculated. These results verify that the proposed method has the advantage of providing an accurate image without the need to increase the number of antennas. The error around the edge points is caused by the error of the numerical derivative.

Next, we show an application example in which the received signals are calculated with FDTD method in a noiseless environment. We extract the quasi wavefront by connecting the peaks of $s\left(X_{\mathrm{T}}, X_{\mathrm{R}}, Y, Z^{\prime}\right)$. Figures 10 and 11 show the estimated image with the conventional and proposed methods, respectively. The total number of estimated points employed in the conventional and proposed methods are 432 and 1920, respectively. Our method provides an accurate image than the conventional method. However, the accuracy is distorted around the target edges compared to that shown in Fig. 9. This is because deformation of the scat- 


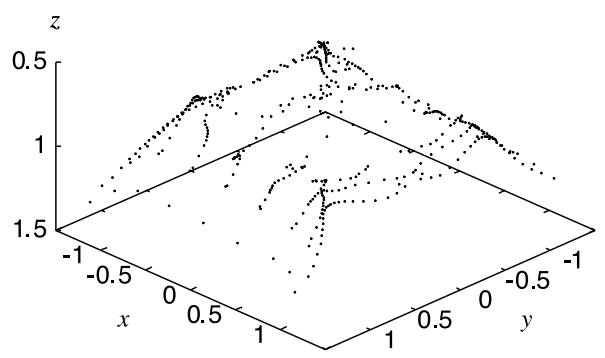

Fig. 10 Estimated image with the conventional method (quasi wavefront is unknown).

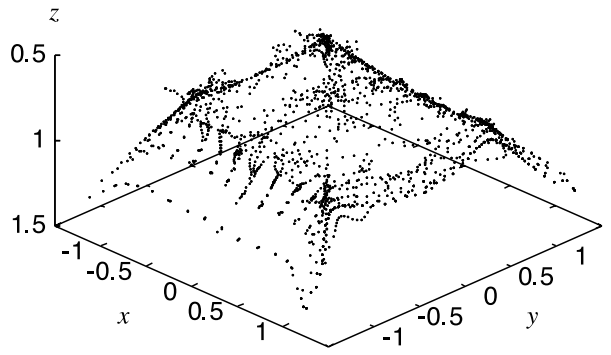

Fig. 11 Estimated image with the proposed method (quasi wavefront is unknown).

tered waveform generates errors in the quasi wavefront. It is our future task to suppress the errors around this region by compensating these distortion, as reported in previous studies [8], [9]. The computational time required for imaging is within $30 \mathrm{msec}$ when using a single Xeon $3.2 \mathrm{GHz}$ processor, which is sufficiently quick for real-time operations.

\subsection{Application Example with the Experiment}

Here, we evaluate the model performance evaluation with an experiment. Figure 12 shows the arrangement of the linear array antennas and the target, as well as the coordinates used in the experiment. We utilize a UWB signal with a center frequency of $3.2 \mathrm{GHz}$ and a $10 \mathrm{~dB}$-bandwidth of $2.0 \mathrm{GHz}$. The antenna has an elliptic polarization whose ratio of the major axis to the minor one is about $17 \mathrm{~dB}$; the direction of the polarimetry is along the $x$ axis. The $3 \mathrm{~dB}$ beam width of the antenna is about $90^{\circ}$. The linear array antennas are set in the vertical direction with 18 antennas. The interval is $100 \mathrm{~mm}$, which corresponds to 1.1 center wavelength of the pulse. The array antennas are scanned along the $y$ axis for $-300 \mathrm{~mm} \leq y \leq 300 \mathrm{~mm}$. The interval between the target and array antennas is about $150 \mathrm{~mm}$ in minimum. The sampling interval is $10.0 \mathrm{~mm}$, and $N_{Y}=61$. The data are coherently averaged 256 times to enhance the $\mathrm{S} / \mathrm{N}$. We preliminarily measure the direct wave from the transmitting antenna without any targets and eliminate this signal from the observed signals with a target in order to obtain the scattered waveform. We observe the transmitted waveform as the reflection from a large specular board that is $1920 \mathrm{~mm}$ in hight and $1180 \mathrm{~mm}$ in width. We utilize the high-frequency relays as switches, where the isolation ratio of each relay is $50 \mathrm{~dB}$

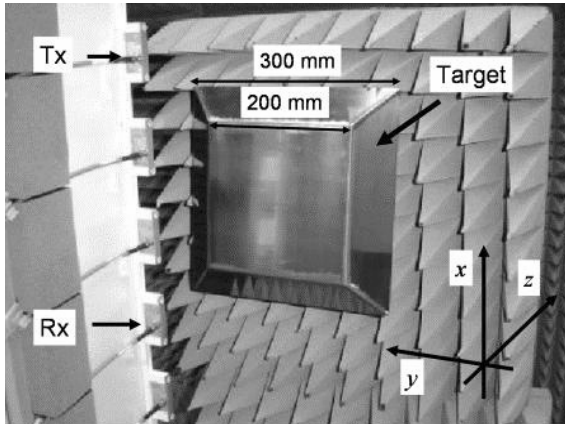

Fig. 12 Linear array antennas and the target.

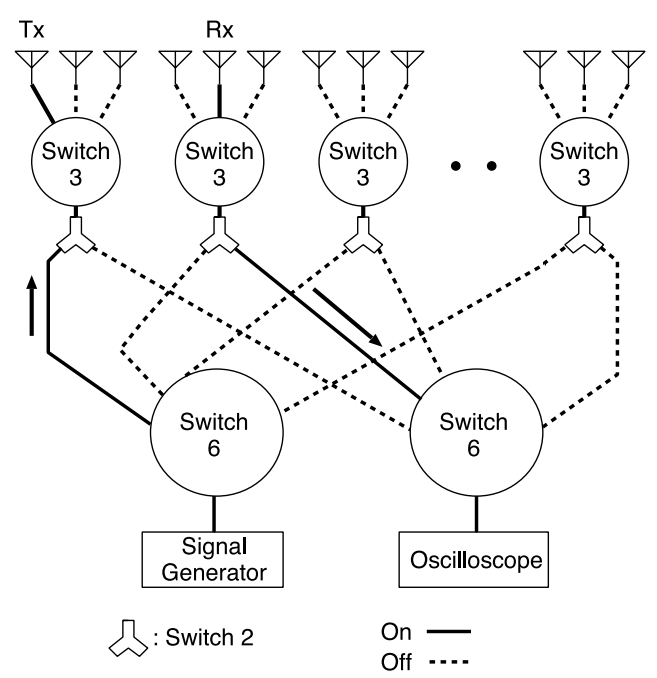

Fig. 13 Arrangement of high-frequency relays and antennas.

and the switching time is within $100 \mathrm{msec}$. We divide the 18 antennas into 6 groups. To simplify the switching system, we do not select transmitting and receiving antennas from the same group. Figure 13 shows the arrangement of the relays and the antennas in the experiment.

We set a metallic hexahedral target made of stainless steel sheeting with a thickness of $3 \mathrm{~mm}$. Figure 14 shows the true target boundary. We utilize 11 antennas set for $-500.0 \mathrm{~mm} \leq x \leq 500.0 \mathrm{~mm}$. Figure 15 shows the output of the matched filter in our experiment, where we set $X_{\mathrm{T}}=100.0 \mathrm{~mm}$ and $X_{\mathrm{R}}=-200.0 \mathrm{~mm}$. The $\mathrm{S} / \mathrm{N}$ in the experiment is $32.0 \mathrm{~dB}$, where we define the $\mathrm{S} / \mathrm{N}$ as the ratio of peak instantaneous signal power to the averaged noise power after applying the matched filter. This also corresponds to the standard deviation $3.0 \times 10^{-3} \lambda$ of the Gaussian random error of the quasi wavefront [10]. The extracted quasi wavefront is smoothed with the Gaussian filter with a correlation length is $0.2 \lambda$. Figure 16 shows the estimated image with the conventional method. Here, the conventional method uses the data for $X_{\mathrm{R}}-X_{\mathrm{T}}=100 \mathrm{~mm}$, which is the minimum interval of the array antennas. The colors of the estimated points represent the estimation error calculated as the distance to the true target boundary. The number of estimated points is 166 . This is because we use only 


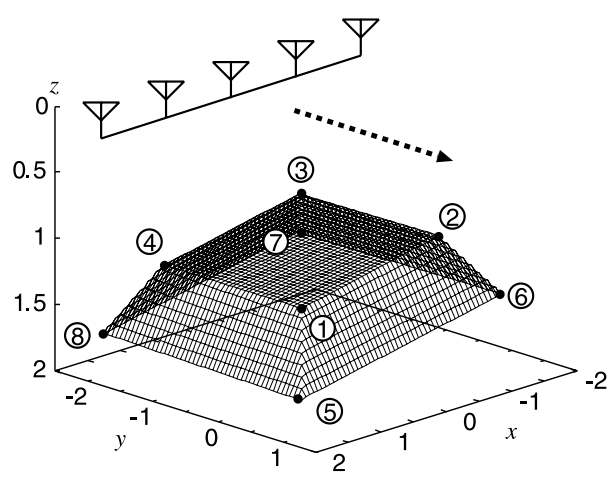

Fig. 14 True target boundary used in the experiment.

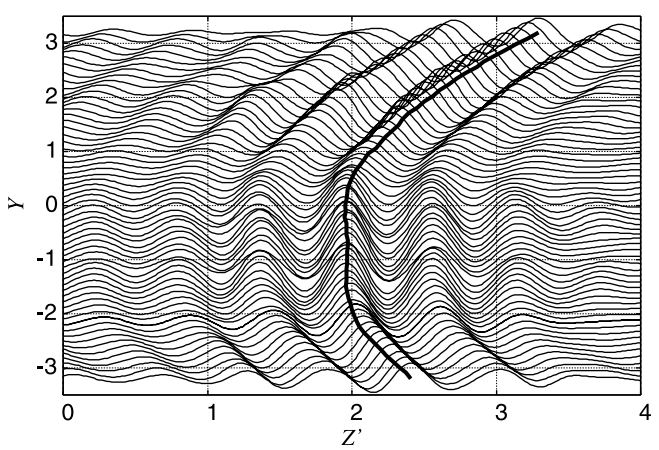

Fig. 15 Example of the output of the matched filter $\left(X_{\mathrm{T}}=100.0 \mathrm{~mm}\right.$, $\left.X_{\mathrm{R}}=-200.0 \mathrm{~mm}\right)$.

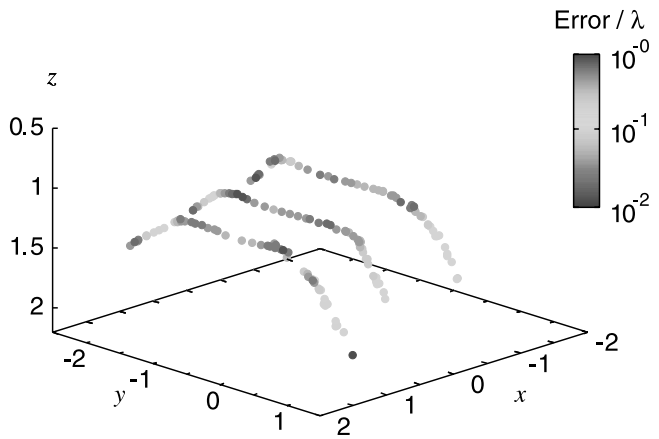

Fig. 16 Estimated image with the conventional method in the experiment.

the data for $\left(X_{\mathrm{T}}, X_{\mathrm{R}}\right)=(-100 \mathrm{~mm}, 0 \mathrm{~mm}),(0 \mathrm{~mm}, 100 \mathrm{~mm})$, $(100 \mathrm{~mm}, 200 \mathrm{~mm})$, which has a sufficient $\mathrm{S} / \mathrm{N}$ and $\mathrm{S} / \mathrm{I}$ to estimate the range. Thus, the number of the estimated points becomes $3 \times N_{Y}$ in maximum, where we remove the points that have imaginary numbers for $z$. The image has insufficient accuracy in the $x$ direction to locate the edges and surface details. Figure 17 shows the estimated image with the proposed method. Our method obtains more accurate image around the target edges and the surface details for the $x$ direction compared to that shown in Fig. 16 . The number of estimated points is 496 . Here, we used the data for $\left(X_{\mathrm{T}}, X_{\mathrm{R}}\right)=(-100 \mathrm{~mm}, 0 \mathrm{~mm}),(0 \mathrm{~mm}, 100 \mathrm{~mm})$, $(100 \mathrm{~mm}, 200 \mathrm{~mm}),(-100 \mathrm{~mm}, 100 \mathrm{~mm}),(0 \mathrm{~mm}, 200 \mathrm{~mm})$, $(100 \mathrm{~mm}, 300 \mathrm{~mm}),(-100 \mathrm{~mm}, 200 \mathrm{~mm}),(0 \mathrm{~mm}, 300 \mathrm{~mm})$,

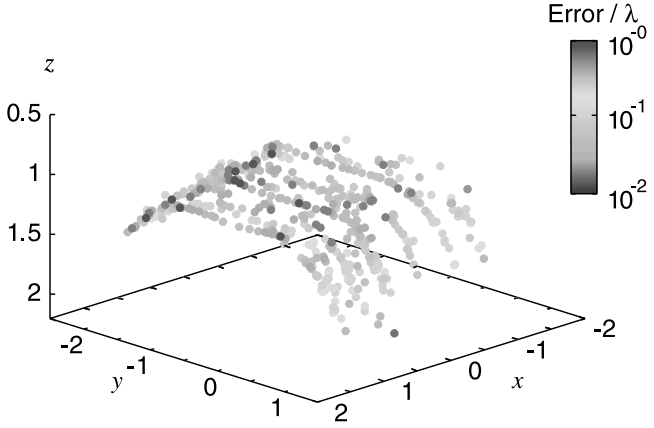

Fig. 17 Estimated image with the proposed method in the experiment.

$(0 \mathrm{~mm}, 400 \mathrm{~mm})$. Thus the number of the proposed method increases to $9 \times N_{Y}$ in maximum. We quantitatively evaluate the error of the two methods with an evaluation value $\epsilon$ that is defined as

$$
\epsilon=\sqrt{\frac{1}{N} \sum_{i=0}^{N} \min _{\boldsymbol{x}}\left\|\boldsymbol{x}-\boldsymbol{x}_{\mathrm{e}}^{i}\right\|^{2},}
$$

where $\boldsymbol{x}$ and $\boldsymbol{x}_{\mathrm{e}}^{i}$ express the location of the true target points and that of the estimated points, respectively. Values of $\epsilon$ with the conventional and proposed methods are $8.8325 \times 10^{-2} \lambda$ and $8.9174 \times 10^{-2} \lambda$, respectively. The estimated image with each method deteriorates around the side of the target due to deformations of the scattered waveform. In addition, Fig. 18 shows the minimum error to the edge points of the target boundary, where the vertical axis in this figure is defined as the minimum distance from the estimated points to each true edge point. The numbers along the horizontal axis correspond to those of the target edges as shown in Fig. 14. Figure 18 confirms that the proposed method is able to determine the edge locations more correctly than the conventional method. Here, the data acquisition time is not the same between the conventional and proposed methods, because the proposed model requires more time for an electrical switching to realize the multi-static measurement. However, the time for the electrical switching, in general, is considerably shorter than that for the mechanical scanning of the antennas with small interval. Thus, if the same accuracy is required, the proposed method remarkably shortens the data acquisition time than the conventional method. It is confirmed that the time for data acquisition with an array antenna scanning is 5 times shorter than that of the mono-static radar system, where the same accuracy is estimated. The calculation time for each method with the received data is $30 \mathrm{msec}$ with a single Xeon $3.2 \mathrm{GHz}$ processor.

However, the estimated image with this method has an instability due to a random noise. Figure 19 shows the projective image on the $x-z$ plane of Fig. 17, where we plot the estimated points in the range for $-0.1 \leq y \leq 0.1$. As shown in this figure, there are some fluctuations around the specular surface. This is because the fluctuation of the quasi wavefront can be enhanced with the derivative operation of IBBST. 


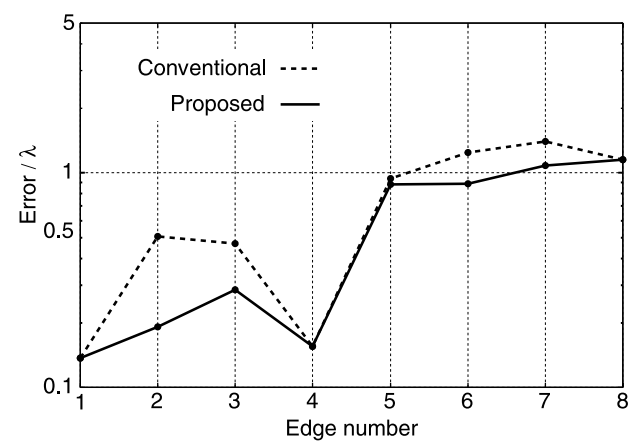

Fig. 18 Estimated error for the target edges.

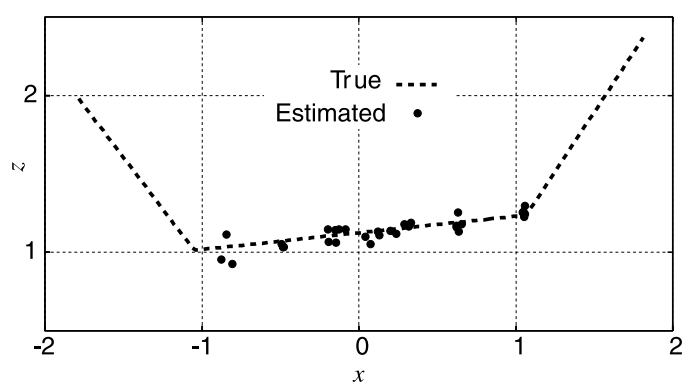

Fig. 19 Projective image on the $x$ - $z$ plane of Fig. $17(-0.1 \leq y \leq 0.1)$.

\section{Discussions on Envelope Method with Array Anten- nas}

We have developed another 2-D imaging algorithm with envelope of circles (Envelope method) [11], and confirmed that it could suppress fluctuations due to derivative operations without data smoothing. This method utilizes the principle that the target boundary can be expressed as the outer or inner envelope of the circles, whose center is $(X, 0)$ and radius is $Z$, in a mono-static radar. It achieves a stable and high-speed 2-D imaging without derivative operations. Furthermore, it can be extended to a bi-static radar, where the target boundary should be expressed as an envelope of ellipses with focuses at $\left(X_{\mathrm{T}}, 0\right)$ and $\left(X_{\mathrm{R}}, 0\right)$ [9]. Thus, this section describes the performance evaluations, when we apply this algorithm to the experimental data with the linear array antennas. Here, we utilize the quasi wavefront extracted with the experimental data, which satisfies $Y=0$, for the 2$\mathrm{D}$ imaging. Figure 20 shows the true and estimated images with Envelope method for the mono-static radar, where the cross section at $y=0$ is illustrated. The number of ellipses for the imaging is 3 . As shown in this figure, the accuracy for the specular region of the target is insufficient. This is because Envelope method approximates the target boundary as the surface of the ellipses. On the contrary, Fig. 21 shows the estimated image with the extended algorithm for bi-static radar. The number of ellipses for the imaging is 5 . The accuracy is enhanced compared to Envelope method for the mono-static radar. These results verify that linear array antennas also enhance the accuracy for the estimated image

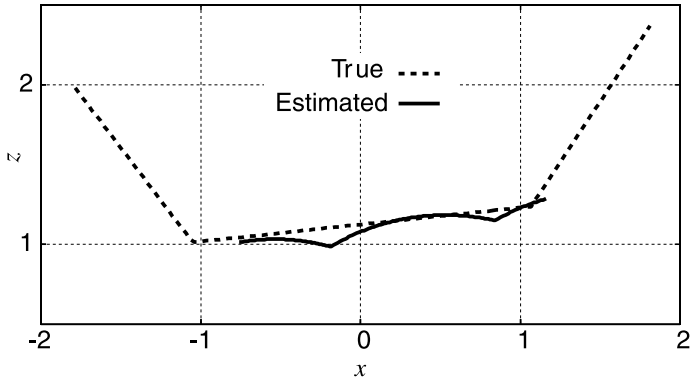

Fig. 20 Cross section image $(y=0)$ with Envelope method for the mono-static radar in the experiment.

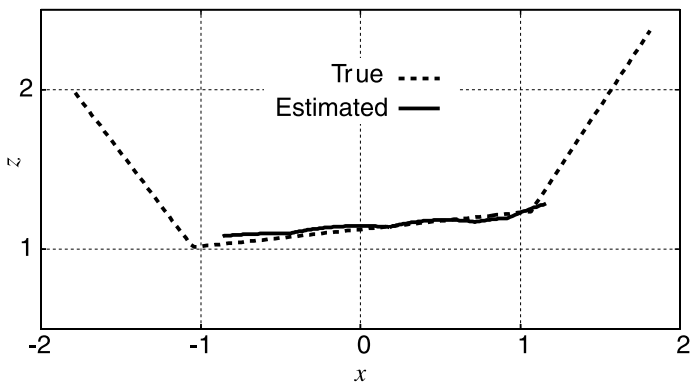

Fig. 21 Cross section image $(y=0)$ with Envelope method for the bi-static radar in the experiment.

with Envelope method. It is our important future task to extend this method to the 3-D model.

\section{Conclusion}

We proposed a fast and accurate imaging algorithm with linear array antennas. The reversible transform BBST for bistatic radars was derived and applied to the array systems. We verified that the proposed method is effective in terms of fast and accurate imaging for 2-D and 3-D problems with numerical simulations. We investigated the performance of the proposed algorithm in experiments that involved linear array antennas. We confirmed that the proposed method improved the accuracy of the image around the edges in the real environment without increasing the number of antennas. It is our future task to suppress the errors around the target edges by compensating the waveform deformations. For discussions, we showed that the UWB array antennas are efficient for another imaging algorithm as Envelope method as well.

\section{Acknowledgment}

This work is supported in part by the 21st Century COE Program (Grant No. 14213201).

\section{References}

[1] C. Chiu, C. Li, and W. Chan, "Image reconstruction of a buried conductor by the genetic algorithm," IEICE Trans. Electron., vol.E84-C, no.12, pp.1946-1951, Dec. 2001. 
[2] T. Takenaka, H. Jia, and T. Tanaka, "Microwave imaging of an anisotropic cylindrical object by a forward-backward time-stepping method," IEICE Trans. Electron., vol.E84-C, no.12, pp.1910-1916, Dec. 2001.

[3] T. Sato, K. Takeda, T. Nagamatsu, T. Wakayama, I. Kimura, and T. Shinbo, "Automatic signal processing of front monitor radar for tunneling machines," IEEE Trans. Geosci. Remote Sens., vol.35, no.2, pp.354-359, 1997.

[4] T. Sato, T. Wakayama, and K. Takemura, "An imaging algorithm of objects embedded in a lossy dispersive medium for subsurface radar data processing," IEEE Trans. Geosci. Remote Sens., vol.38, no.1, pp.296-303, 2000.

[5] T. Sakamoto and T. Sato, "A target shape estimation algorithm for pulse radar systems based on boundary scattering transform," IEICE Trans. Commun., vol.E87-B, no.5, pp.1357-1365, May 2004.

[6] T. Sakamoto and T. Sato, "A phase compensation algorithm for highresolution pulse radar systems," IEICE Trans. Commun., vol.E87-B, no.6, pp.1631-1638, June 2004.

[7] T. Sakamoto, "A fast algorithm for 3-dimensional imaging with UWB pulse radar systems," IEICE Trans. Commun., vol.E90-B, no.3, pp.636-644, March 2007.

[8] S. Kidera, T. Sakamoto, T. Sato, and S. Sugino, "An accurate imaging algorithm with scattered waveform estimation for UWB pulse radars," IEICE Trans. Commun., vol.E89-B, no.9, pp.2588-2595, Sept. 2006

[9] S. Kidera, T. Sakamoto, and T. Sato, "A robust and high-resolution imaging algorithm with waveform estimation for UWB pulse radars," IEICE Trans. Commun., vol.E90-B, no.6, pp.1487-1494, June 2007.

[10] T. Sakamoto, "A 2-D image stabilization algorithm for UWB pulse radars with fractional boundary scattering transform," IEICE Trans Commun., vol.E90-B, no.1, pp.131-139, Jan. 2007.

[11] S. Kidera, T. Sakamoto, and T. Sato, "A robust and fast imaging algorithm with an envelope of circles for UWB pulse radars," IEICE Trans. Commun., vol.E90-B, no.7, pp.1801-1809, July 2007.

\section{Appendix A: Derivation of Eqs. (2) and (3).}

First, let us derive Eq. (3). As shown in Fig. 3, the target boundary $(x, z)$ should be on an ellipse as

$$
\frac{(x-X)^{2}}{Z^{2}}+\frac{z^{2}}{Z^{2}-d^{2}}=1,
$$

where $Z>d>0$ holds. We define $G(x, z ; X, Z, d)$ as

$$
\begin{aligned}
G(x, z ; X, Z, d)= & \left(Z^{2}-d^{2}\right)(x-X)^{2} \\
& +Z^{2} z^{2}-Z^{2}\left(Z^{2}-d^{2}\right) .
\end{aligned}
$$

Thus, Eq. (A. 1) is expressed as

$$
G(x, z ; X, Z, d)=0 .
$$

Moreover, the target boundary $(x, z)$ exists on an envelope of ellipses in Eq. (A. 1) with parameter $X$ as

$$
\partial G(x, z ; X, Z, d) / \partial X=0,
$$

Eliminating $z$ in Eqs. (A. 3) and (A. 4), we obtain

$$
d^{2} Z_{X}(x-X)^{2}-Z\left(Z^{2}-d^{2}\right)(x-X)-Z^{4} Z_{X}=0,
$$

where $Z_{X}=\partial Z / \partial X$ is defined. If $d \neq 0$ and $Z_{X} \neq 0$ holds, $x$ is expressed as

$$
x=X-\frac{2 Z^{3} Z_{X}}{Z^{2}-d^{2} \pm \sqrt{\left(Z^{2}-d^{2}\right)^{2}+4 d^{2} Z^{2} Z_{X}^{2}}} .
$$

We define the two solutions as $x_{+}$and $x_{-}$, respectively, where the subscript of the solution corresponds to the mark of the square root. By taking $d \rightarrow 0$ for the absolute value of $x_{+}$and $x_{-}$, we obtain

$$
\begin{aligned}
& \lim _{d \rightarrow 0}\left|x_{+}\right|=\left|X-Z Z_{X}\right|, \\
& \lim _{d \rightarrow 0}\left|x_{-}\right|=\infty .
\end{aligned}
$$

With the condition that $x$ converges to the finite for $d \rightarrow 0$, $x$ is given as

$$
x=X-\frac{2 Z^{3} Z_{X}}{Z^{2}-d^{2}+\sqrt{\left(Z^{2}-d^{2}\right)^{2}+4 d^{2} Z^{2} Z_{X}^{2}}}
$$

Equation (A.9) corresponds to the solutions, where we set $Z_{X}=0$ and $d=0$ in Eq. (A. 5). Solving Eq. (A- 1) for $z$, Eq. (3) is derived.

Equation (2) can be derived in a similar manner. $g(X, Z ; x, z, d)$ is defined as

$$
\begin{aligned}
g(X, Z ; x, z, d)= & \left(Z^{2}-d^{2}\right)(x-X)^{2} \\
& +Z^{2} z^{2}-Z^{2}\left(Z^{2}-d^{2}\right) .
\end{aligned}
$$

The point $(X, Z)$ should be on an envelope of the curves in Eq. (A.10) with parameter $x$. Thus, $(X, Z)$ satisfies

$$
\begin{aligned}
& g(X, Z ; x, z, d)=0, \\
& \partial g(X, Z ; x, z, d) / \partial x=0 .
\end{aligned}
$$

Eq. (2) is derived by solving the above two equations.

We prove a reversibility between Eqs. (2) and (3). We define $z^{\prime}$ as an arbitrary single-valued function of $x$, which is differentiable. $(X, Z)$ is defined with BST as $X=x+$ $z^{\prime} z^{\prime}, Z=z^{\prime} \sqrt{1+z_{x}^{\prime 2}}$. The reversibility between $\left(x, z^{\prime}\right)$ and $(X, Z)$ is proved in [5], and

$$
\begin{aligned}
& G\left(x, z^{\prime} ; X, Z, 0\right)=g\left(X, Z, x, z^{\prime}, 0\right)=0, \\
& \partial G\left(x, z^{\prime} ; X, Z, 0\right) / \partial X=0, \\
& \partial g\left(X, Z ; x, z^{\prime}, 0\right) / \partial x=0,
\end{aligned}
$$

should hold. Here, we define $z$ as $z=\frac{\sqrt{Z^{2}-d^{2}}}{Z} z^{\prime}$, where $d$ is constant and $Z>d>0$ holds. Substituting $z$ into Eqs. (A. 13), (A. 14) and (A. 15), we obtain

$$
\begin{aligned}
& \frac{(x-X)^{2}}{Z^{2}}+\frac{z^{2}}{Z^{2}-d^{2}}=1, \\
& d^{2} Z_{X}(x-X)^{2}-Z\left(Z^{2}-d^{2}\right)(x-X), \\
& \quad-Z^{4} Z_{X}=0 \\
& \left(Z^{2}-d^{2}\right)(x-X)+Z^{2} z z_{x}=0 .
\end{aligned}
$$

These three equations correspond to Eqs. (A. 3), (A.4) and (A. 12), respectively. Accordingly, $(X, Z)$ and $(x, z)$ should satisfy Eqs. (2) and (3). In addition, $z^{\prime}=\frac{Z}{\sqrt{Z^{2}-d^{2}}} z$ holds, and $(x, z)$ and $\left(x, z^{\prime}\right)$ are reversible. Thus, the reversibility between $(x, z)$ and $(X, Z)$ should hold. Figure A. 1 shows the relationship among $\left(x, z^{\prime}\right),(X, Z)$, and $(x, z)$. 


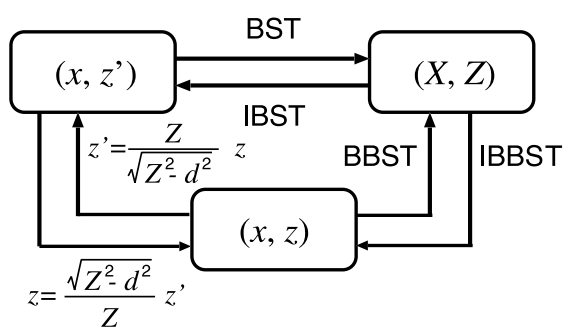

Fig. A· 1 Relationship between $(x, z),\left(x, z^{\prime}\right)$ and $(X, Z)$.

\section{Appendix B: Derivation of Eqs. (4) and (5).}

We derive Eq. (5) as follows. The point on the target boundary $(x, y, z)$ should fall on the ellipsoid as

$$
\frac{(x-X)^{2}}{Z^{2}}+\frac{(y-Y)}{Z^{2}-d^{2}}+\frac{z^{2}}{Z^{2}-d^{2}}=1,
$$

where $Z>d>0$ holds. Here we define the function $G(x, y, z ; X, Y, Z, d)$ as

$$
\begin{aligned}
G(x, y, z ; X, Y, Z, d)= & \left(Z^{2}-d^{2}\right)(x-X)^{2}+Z^{2}(y-Y)^{2} \\
& +Z^{2} z^{2}-Z^{2}\left(Z^{2}-d^{2}\right) . \quad(\text { A } \cdot 20)
\end{aligned}
$$

Equation $(\mathrm{A} \cdot 28)$ is expressed as

$$
G(x, y, z ; X, Y, Z, d)=0 .
$$

The target boundary should be on an envelope of ellipsoids with the parameters $X$ and $Y$. Thus,

$$
\begin{aligned}
& \partial G(x, y, z ; X, Y, Z, d) / \partial X=0, \\
& \partial G(z, y, z ; X, Y, Z, d) / \partial Y=0,
\end{aligned}
$$

hold. Eliminating $y$ and $z$ in Eqs. (A. 21) and (A. 22), the solution for $x$ is expressed as

$$
x=X-\frac{2 Z^{3} Z_{X}}{Z^{2}-d^{2}+\sqrt{\left(Z^{2}-d^{2}\right)^{2}+4 d^{2} Z^{2} Z_{X}^{2}}} .
$$

Here we specify the subscript of the solution using a similar approach to that used in Appendix A. In addition, eliminating $z$ in Eqs. (A.21) and (A.23), $y$ is expressed as

$$
y=Y+Z_{Y}\left\{d^{2}(x-X)^{2}-Z^{4}\right\} / Z^{3}
$$

With Eq. (A. 5), $z$ is expressed as

$$
z=\sqrt{Z^{2}-d^{2}-(y-Y)^{2}-\frac{\left(Z^{2}-d^{2}\right)(x-X)^{2}}{Z^{2}}} .
$$

Thus, Eq. (5) is derived. Equation (4) can be derived, where we define $g(X, Y, Z ; x, y, z, d)$ as

$$
\begin{aligned}
g(X, Y, Z ; x, y, z, d)= & \left(Z^{2}-d^{2}\right)(x-X)^{2}+Z^{2}(y-Y)^{2} \\
& +Z^{2} z^{2}-Z^{2}\left(Z^{2}-d^{2}\right) .
\end{aligned}
$$

$(X, Y, Z)$ should satisfy the following conditions,

$$
g(X, Y, Z ; x, y, z, d)=0,
$$

$$
\begin{aligned}
& \partial g(X, Y, Z ; x, y, z, d) / \partial x=0, \\
& \partial g(X, Y, Z ; x, y, z, d) / \partial y=0 .
\end{aligned}
$$

The reversibility between $(x, y, z)$ and $(X, Y, Z)$ is proved using the similar way of that in Appendix A.

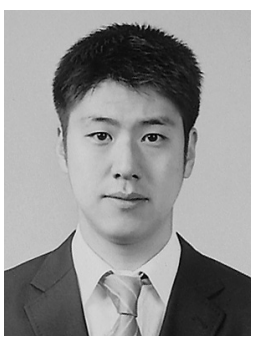

Shouhei Kidera received the B.E. degree from Kyoto University in 2003 and the M.I. degree at Graduate School of Informatics, Kyoto University in 2005 . He is currently studying for the Ph.D. degree at Graduate School of Informatics, Kyoto University. His current research interest is in signal processing for UWB pulse radars. He is a member of the IEEE.

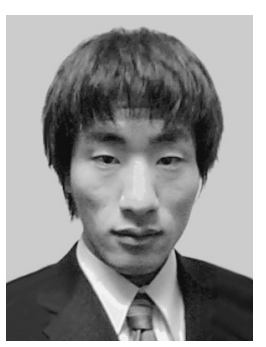

Yusuke Kani received the B.E. degree from Kyoto University in 2007. He is currently studying for the M.I. degree at Graduate School of Informatics, Kyoto University. His current research interest is in signal processing for UWB pulse radars.

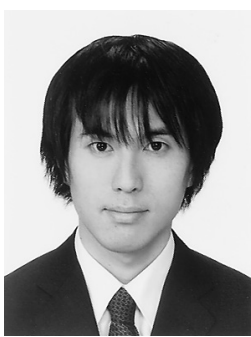

Takuya Sakamoto was born in Nara, Japan in 1977. Dr. Sakamoto received his B.E. degree from Kyoto University in 2000, and his M.I. and Ph.D. degrees from Graduate School of Informatics, Kyoto University in 2002 and 2005, respectively. $\mathrm{He}$ is a research associate in the Department of Communications and Computer Engineering, Graduate School of Informatics, Kyoto University. His current research interest is in signal processing for UWB pulse radars. $\mathrm{He}$ is a member of the IEEJ and the IEEE.

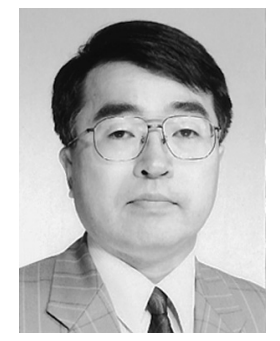

Toru Sato received his B.E., M.E., and $\mathrm{Ph} . \mathrm{D}$. degrees in electrical engineering from Kyoto University, Kyoto, Japan in 1976, 1978, and 1982, respectively. He has been with Kyoto University since 1983 and is currently a Professor in the Department of Communications and Computer Engineering, Graduate School of Informatics. His major research interests have been system design and signal processing aspects of atmospheric radars, radar remote sensing of the atmosphere, observations of precipitation using radar and satellite signals, radar observation of space debris, and signal processing for subsurface radar signals. Dr. Sato was awarded Tanakadate Prize in 1986. He is a member of the Institute of Electronics, Information, and Communication Engineers of Japan, the Society of Geomagnetism and Earth, Planetary and Space Sciences, the Japan Society for Aeronautical and Space Sciences, the Institute of Electrical and Electronics Engineers, and the American Meteorological Society. 